\title{
Malignant causes of fever of unknown origin
}

\author{
Authors: Vanessa Foggo ${ }^{A}$ and Jamie Cavenagh ${ }^{B}$
}

The presence of fever in malignancy usually indicates infection, though transfusion, thrombosis and drugs are also culprits. However, particularly in some tumour types, fever can also be a paraneoplastic syndrome, caused by the malignancy itself. This can be a difficult diagnosis to establish and presents a therapeutic challenge to the physician when the underlying malignancy is not easily treated.

\section{Introduction}

The mechanisms by which malignancies induce fever are not fully understood. The release of pyrogenic cytokines either directly from tumour cells or from macrophages responding to tumour are likely to play a major role, particularly interleukin (IL)-1, IL- 6 and tumour necrosis factor (TNF) $\alpha$. Cytokines induce prostaglandin E2 which acts on the hypothalamus, causing a change in the thermostatic set point. Although cancer is often a concern in fever of unknown origin (FUO), malignancy appears to account for a decreasing proportion of those investigated. ${ }^{1}$ In 1961, 19\% of FUO were attributed to cancer and by 2007 this had fallen to $7 \%$. Neoplastic fevers are now most commonly encountered in the setting of febrile patients with a known malignancy and presents a diagnostic challenge in differentiating whether fever is attributable to infection, therapy or disease.

Unfortunately there is no fever pattern pathognomonic of cancer. Classically, neoplastic fever may be less associated with rigors, tachycardia and hypotensive episodes than other causes. Fevers may be only partially relieved by paracetamol and may respond better to nonsteroidal anti-inflammatory drug (NSAIDs). Cyclical fever patterns may occur. The most well known of these is Pel-Ebstein fever associated with Hodgkin's lymphoma. ${ }^{2}$ However, the pattern of a week of high fevers, followed by an afebrile period of similar duration, is rare, and both its specificity for Hodgkin's lymphoma and its existence as an entity at all are contentious. ${ }^{2}$

\section{Naproxen test}

An interesting series of small studies published by Chang and Gross $^{3}$ in 1984 observed that neoplastic fever was suppressed by naproxen. Defervescence occurred in 14 of 15 patients with

Authors: ASpR haematology, Royal London Hospital, London, UK; Bprofessor of haematology, St Bartholomew's Hospital, London, UK neoplastic fever in contrast to none of the 5 patients with fever due to infection. Two patients with connective tissue disease had partial lysis of fever. In patients with neoplastic fever, the fevers resolved within 24 hours and patients remained afebrile as long as they continued on naproxen. This led to the description of a naproxen test and the suggestion of diagnostic criteria for neoplastic fever. ${ }^{4}$

Follow-up data of larger groups of cancer patients has supported that the naproxen test has a high degree of sensitivity and specificity, with a high positive predictive value. ${ }^{5,6}$ Particularly when clinical suspicion is high, this may provide an effective aid in differentiating neoplastic from infectious fever in cancer patients in whom naproxen is not contraindicated (see Box 1).

Fever can serve an important purpose in alerting clinicians to the presence of an underlying malignancy. However, in the investigation of oncology patients with fever, it should remain a diagnosis of exclusion. Once the diagnosis of neoplastic fever is established, clearly the priority is to treat the malignancy. When that is not possible, naproxen may be able to provide some symptom relief. There is some evidence that other NSAIDs, including indomethacin and diclofenac, are also effective. ${ }^{7}$

\section{Cancers most associated with neoplastic fever}

Among cancers, the common culprits for neoplastic or paraneoplastic fever include: Castleman's disease, Hodgkin's

\section{Key points}

Presence of fever in patients with cancer usually indicates infection.

The first priority should be to treat underlying infection and only once infection is excluded should other causes of fever be considered.

Malignancy is well known to cause fever, particularly in association with certain tumour types.

In cancer patients with fever of unknown origin, the naproxen test may help diagnose neoplastic fever (see Box 1).

NSAIDs, and other anti-inflammatory agents may help control symptoms of fever until the cancer can be treated.

KEYWORDS: Neoplastic fever, fever, FUO, malignancy 
and non-Hodgkin's lymphoma, renal cell carcinoma, hepatocellular carcinoma, acute myeloid leukaemia, hairy cell leukaemia, glioblastoma multiforme, blast crisis of chronic myelogenous leukemia, ovarian cancer and atrial myxoma. Neoplastic fever has differing manifestations and significance depending on the underlying tumour type, and may hold prognostic value.

\section{Castleman's disease}

This rare lymphoproliferative disorder can be localised or multicentric. In both types there can be hyperactivation of the immune system, hypersecretion of cytokines and proliferation of $\mathrm{B}$ and T cells. The condition, when seen in patients with HIV is associated with human herpes virus (HHV)-8 infection. Systemic symptoms include fever, night sweats, fatigue, anorexia and cachexia. Patients can have lymphadenopathy, hepatosplenomegaly and anaemia. The acute phase proteins are increased, including CRP and fibrinogen. IL-6 is the most commonly elevated cytokine and is pivotal to the pathophysiology of Castleman's disease. It is the excess production of IL-6, which appears to lead to the constitutional symptoms, as well as proliferative features of the disease. This may result from dysregulated production by tumour cells, or in $\mathrm{HHV}-8$-associated disease production of a viral homolog of IL- $6 .{ }^{8-10}$

Unicentric Castleman's disease is treated by surgical resection. The poor prognosis of multicentic Castlemans has been improved by the introduction of anti-IL- 6 agents. In a recent randomised controlled trial, ${ }^{11}$ patients who received siltuximab demonstrated durable tumour and symptomatic responses. As use of this drug widens, it will be interesting to see it has therapeutic benefit in other cancers associated with systemic inflammation.

\section{Lymphoma}

High levels of IL-6 and IL-10 have been observed in lymphomas, and the presence of B symptoms correlates with serum levels of IL- $6 .^{12}$

\section{Box 1. Diagnostic criteria for neoplastic fever.}

Temperature over $37.8^{\circ} \mathrm{C}$ at least once each day.

$>$ Duration of fever over two weeks.

$>$ Lack of evidence of infection on:

> physical examination

> laboratory examinations eg sputum smears or cultures, cultures of blood, urine, stool, bone marrow, spinal fluid, pleural fluid and discharge from local lesions

> imaging studies eg chest radiograph and CT scans of the head, abdomen, and pelvis.

> Absence of allergic mechanisms eg drug allergy, transfusion reaction, and radiation or chemotherapeutic drug reaction.

> Lack of response of fever to an empiric, adequate antibiotic therapy for at least seven days.

> Prompt, complete lysis of fever by the naproxen test ${ }^{a}$ with sustained normal temperature while receiving naproxen.

anaproxen 375 mg (by mouth) every 12 hours for three days. Reproduced with permission. ${ }^{4}$
B symptoms, including fever, night sweats and weight loss, are present in approximately $25 \%$ of patients with Hodgkin's lymphoma. B symptoms are associated with a worse prognosis, so are included in disease staging and impact on choice of treatment regimes.

\section{Haemophagocytic lymphohistiocytosis}

Haemophagocytic lymphohistiocytosis (HLH) is a rare severe inflammatory disease characterised by high fevers and pancytopenia. Other features include lymphadenopathy, hepatosplenomegaly, rash and neurological symptoms. Evidence of haemophagocytosis (histiocytes eating other blood cells) is found on bone marrow or lymph node biopsy. The disease can be inherited or associated with infection, rheumatological disorders or malignancy, particularly T- or NK-cell leukaemias and lymphomas. Its genetic and immunological basis is increasingly well understood. Genetic defects of cytotoxicity and/or acquired absence of NK-cell function leads to immune activation and inflammatory cytokine production, with consequent disease development. ${ }^{13,14}$ Patients have strikingly high levels of inflammatory cytokines whose production may drive fevers and other clinical features. Treatment strategies combine immunotherapy and chemotherapy to achieve remission of symptoms, often as a bridge to bone marrow transplantation.

\section{Renal cell carcinoma}

In some series, up to $20 \%$ of patients with renal cell carcinoma (RCC) present with fever, and systemic symptoms, including fever, weight loss, hypertension and paraneoplastic phenomena, do seem to correlate with tumour size. In a previous study, Song ${ }^{15}$ reported a 1.54 increased odds of systemic symptoms with every $1 \mathrm{~cm}$ increase in tumour size. It seems logical that larger tumours might produce or provoke greater concentrations of inflammatory mediators.

In patients with RCC, increased serum IL-6 levels have been associated with neoplastic fever and also with advanced stage, poor performance status and decreased responsiveness to immunotherapy. ${ }^{16-18}$

\section{Therapy}

In most cases, treatment targets the underlying neoplastic condition, however this is not always successful or feasible. Fever can be a distressing symptom of advanced malignancy in the palliative setting. The metabolic demands associated with frequent generation of fever can contribute to cancer cachexia. In palliative care, we frequently attempt to control the symptoms with paracetamol, NSAIDS and corticosteroids. Steroids such as dexamethasone inhibit a variety of cytokines, including IL-2, IL-4, IL-6, IL-10, IL-12, interferon (IFN) $\gamma$ and TNF $\alpha$. Although a blunt tool with many side effects, they are often used in this setting, particularly when paracetamol or NSAIDS are ineffective.

\section{Novel therapies}

One interesting development has arisen out of the discovery that thalidomide has a marked effect on fever associated with 
leprosy. ${ }^{19}$ Thalidomide is an immunomodulatory agent with a wide spectrum of activity. It acts on the protein cereblon and its interaction with the enzyme complex formed by cereblon results in the drug's antiproliferative and teratogenic effects. Thalidomide enhances the release of IL-2 and IFN from activated $\mathrm{T}$ cells, inhibits the immunosuppressive activity of regulatory $\mathrm{T}$ cells and increases NK-cell mediated cytotoxicity. It inhibits the release of TNF $\alpha$ and other cytokines including IL- $6 .{ }^{20}$ This may explain the potential anti-pyretic effect. Thalidomide has been found to be effective in cancer cachexia, chronic nausea, insomnia, profuse sweating and pain. ${ }^{21}$ In the context of advanced malignancy, the catastrophic teratogenetic effects are irrelevant and other side effects including neuropathy may be less of a concern. However, thalidomide is a relatively expensive drug which needs careful control and regulation. It may prove inaccessible to many patients in this setting until proven to offer benefit.

Hopes for future treatments rest on better understanding the pathophysiology of malignancy-induced fever and exploiting unique features to improve symptom control.

\section{References}

1 Horowitz HW. Fever of unknown origin or fever of too many origins? N Engl J Med 2013;368:197-9.

2 Schattner A, Keshet N. Pel-Ebstein cyclic fever: not just lymphoma. Am J Med 2010;123:e3.

3 Chang JC, Gross HM. Utility of naproxen in the differential diagnosis of fever of undetermined origin in patients with cancer. Am J Med 1984;76:597-603.

4 Zell JA, Chang JC. Neoplastic fever: a neglected paraneoplastic syndrome. Support Care Cancer 2005;13:870-7.

5 Johnson M. Neoplastic fever. Palliat Med 1996;10:217-24.

6 Browder AA, Huff JW, Petersdorf RG. The significance of fever in neoplastic disease. Ann Intern Med 1961;55:932-42.

7 Rabon P, Murray KM. Role of nonsteroidal antiinflammatory drugs in the differential diagnosis of neoplastic fevers versus fevers of infectious etiology. J Pharm Tech 1995;11:211-3.

8 Dispenzieri A, Armitage JO, Loe MJ et al. The clinical spectrum of Castleman's disease. Am J Hematol 2012;87:997-1002.
9 Casper C. The aetiology and management of Castleman disease at 50 years: translating pathophysiology to patient care. Br J Haematol 2005;129:3-17.

10 van Rhee F, Stone K, Szmania S et al. Castleman disease in the 21st century: an update on diagnosis, assessment, and therapy. Clin $A d v$ Hematol Oncol 2010;8:486-98.

11 van Rhee F, Wong RS, Munshi N et al. Siltuximab for multicentric Castleman's disease: a randomised, double-blind, placebocontrolled trial. Lancet Oncol 2014;15:966-74.

12 Seymour JF, Talpaz M, Cabanillas F et al. Serum interleukin-6 levels correlate with prognosis in diffuse lareg cell lymphoma. J Clin Oncol 1995;13:575-82.

13 Jordan MB, Allen CE, Weitzman S at al. How I treat hemophagocytic lymphohistiocytosis. Blood 2011;118:4041-52.

14 Jordan MB, Hildeman D, Kappler J, Marrack P. An animal model of hemophagocytic lymphohistiocytosis (HLH): CD8+ T cells and interferon gamma are essential for the disorder. Blood 2004;104:735-43.

15 Song J, Tanagho Y, Bhayani S, Figenshau R. Factors predictive of symptomatic presentation in renal cell carcinoma. J Endourol 2013;27:0892-7790.

16 Kim HL, Belldegrun AS, Freitas DG et al. Paraneoplastic signs and symptoms of renal cell carcinoma: implications for prognosis. J Urol 2003;170:1742-6.

17 Blay JY, Negrier S, Combaret V et al. Serum level of interleukin 6 as a prognosis factor in metastatic renal cell carcinoma. Cancer Res 1992; 52:3317-22.

18 Blay JY, Rossi JF, Wijdenes J et al. Role of interleukin-6 in the paraneoplastic inflammatory syndrome associated with renal-cell carcinoma. Int J Cancer 1997;72:424-30.

19 Sampaio EP, Kaplan G, Miranda A et al. The influence of thalidomide on the clinical and immunologic manifestation of erythema nodosum leprosum. J Infect Dis 1993;168(2):408-14.

20 Krönke J, Udeshi ND, Narla A et al. Mechanism of lenalidomide activity in multiple myeloma. Science 2014;343:301-5.

21 Peuckmann V, Fisch M, Bruera E. Potential novel uses of thalidomide. Drugs 2000;60:273-92.

Address for correspondence: Dr V Foggo, Department of Haematology, The Royal London Hospital, Whitechapel Road, London E1 1BB, UK.

Email: vanessa.foggo@bartshealth.nhs.uk 